\title{
Emergency Room Thoracotomy (ERT): A Retrospective Audit of Results
}

\author{
Barbaro I. Monzon ${ }^{1 *}$, Maria del Carmen Ortega ${ }^{2}$, Jacques Goosen $^{3}$, \\ Dietrich Doll ${ }^{4}$, Maeyane Stephen Moeng ${ }^{5}$
}

Received: 27 July 2020 / Accepted: 14 August 2020 / Published online: 20 January 2021

(C) The Author(s) 2021. This article is published with open access at https://journal.astes.org.al

\begin{abstract}
Background: An Emergency Room Thoracotomy (ERT) is a resource-intensive, high-risk procedure in which rapid decision-making is essential. In a resource-constrained system, identification of the group of patients that could achieve the best outcome will avoid futile use. Incorporating physiological and metabolic parameters at the time of arrival to the emergency department into the management algorithm may assist with better patient selection and could improve outcomes.
\end{abstract}

Material and Methods: A retrospective review of the results of subjects who underwent Emergency Room Thoracotomy at a Level 1 Academic Trauma Center over a 13-year period (01 January 2005 to 31 December 2017) was conducted. Mechanism of injury, physiological and metabolic parameters, anatomical injuries, Injury Severity Score (ISS), calculated Revised Trauma score (cRTS), volume and type of fluids administered, and mortality were analyzed comparing survivors and non-survivors.

Results: One hundred and ten $(\mathrm{n}=110)$ patients underwent ERT during the study period. Variables such as the mechanism of injury, physiological and metabolic parameters, type, and volume of fluids administered did not show any statistically significant influence in the final outcome. Penetrating cardiac and chest trauma had better survival ( $40.6 \%$ and $20 \%$ respectively) compared to those with thoraco-abdominal, abdominal, pelvic, and femoral vessel trauma. Overall survival was $21,8 \%$.

Conclusions: In a resource-constrained environment an Emergency Room Thoracotomy should be performed in patients with a thoracic injury, especially cardiac, to achieve the best possible outcome.

Keywords: Trauma, ERT, Mortality

Original article, no submission or publication in advance or in parallel

* Corresponding author:

Barbaro I. Monzon, MD

$\bowtie$ bignaciomonzon@gmail.com

1 Department of Surgery, Steve Biko Academic Hospital, School of Medicine, Faculty of Health Sciences University of Pretoria, Gezina 0031, Trauma Unit Charlotte Maxeke Johannesburg Academic Hospital, Department of Surgery, School of Clinical Medicine, Faculty of Health Sciences, University of the Witwatersrand, Jubilee Road Parktown, Johannesburg, 2196, South Africa. SOUTH AFRICA

2 Maria del Carmen Ortega MD Anaesthetist in Private Practice, Johannesburg SOUTH AFRICA.

3 Jacques Goosen MBChB Trauma Unit Charlotte Maxeke Johannesburg Academic Hospital, Department of Surgery, School of Clinical Medicine, Faculty of Health Sciences, University of the Witwatersrand, Jubilee Road, Parktown, Johannesburg, 2196, SOUTH AFRICA.

4 Dietrich Doll MD PhD Department of Procto-Surgery, Catholic Clinics Oldenburger Münsterland GmbH, St. Marys Hospital, Marienstraße 6-8, D-49377 Vechta, Germany and Department of Surgery, School of Clinical Medicine, Faculty of Health Sciences, University of the Witwatersrand, Jubilee Road Parktown, Johannesburg, 2196, SOUTH AFRICA.

5 Maeyane Stephen Moeng MBChB Trauma Unit Charlotte Maxeke Johannesburg Academic Hospital, Department of Surgery, School of Clinical Medicine, Faculty of Health Sciences, University of the Witwatersrand, Jubilee Road Parktown, Johannesburg, 2196, SOUTH AFRICA.

\section{Introduction}

The major causes of early trauma deaths are severe traumatic brain injury and irreversible haemorrhagic shock secondary to uncontrolled bleeding [1-4].

Early, aggressive control of haemorrhage, with rational use of blood and goal directed management of coagulation and limited use of crystalloid fluids improves survival [2$11]$.

The improvements in the emergency medical rescue systems that have occurred in recent years increases access to hospital care, therefore a larger number of otherwise "fatally" injured bleeding patients arrive to emergency departments with significant physiological and metabolic deterioration. Efforts to salvage these patients often involve desperate measures to control haemorrhage and restore tissue perfusion.

Opening the chest in the emergency department to relieve cardiac tamponade, suture a lacerated heart, crossclamp the supra-diaphragmatic thoracic Aorta to reduce the 
rate of bleeding in the abdomen and pelvis, control bleeding from the lung and mediastinal great vessels and perform open cardiac massage for traumatic cardiac arrest have been part of the surgical arsenal for many years. [1,12-18]

The current scientific literature clearly supports the use of ERT for injuries in the chest and especially the heart where the best outcomes are obtained [1,13-33]. However, there are ongoing debates about its role in blunt trauma [24-33]. Futile use is a large concern in overloaded underfunded trauma systems and centers $[19,20,21,22]$. The risk of performing an ERT in blunt trauma victims has to be weighed against the expected benefit for the individual patient in these situations [32,33].

The Resuscitative Endovascular Balloon Occlusion (REBOA) is emerging as an alternative to cross-clamping of the Aorta during ERT. REBOA seems to benefits those patients with abdominal and pelvic bleeding, however, this procedure is not yet widely available and offers no benefit in chest trauma. [35,36]

The caveat of ERT is the high mortality rate, poor neurological outcomes, risk of complications and hazards present during ERT, especially the transmission of infectious diseases to health care personnel, as well as the high costs involved in the management of these very ill patients $[1,13$ 33] These factors have prompted investigators to analyze the outcomes of ERT to identify the patient population with the best results.

The aim of this study was to analyze the clinical characteristic of the subjects that underwent ERT in our hospital and their potential influence in their survival.

We hypothesize that in our resources-constrained health care system an ERT should be directed only to those patients with the best chances of survival and that incorporating physiological and metabolic data to the decision-making algorithm could assist in selecting the patients for the procedure. The experience obtained could be relevant to centres and hospitals that have resource limitations similar to our institution.

\section{Material and Method}

The study describes the findings of the results of adult patients who underwent ERT between $1^{\text {st }}$ January 2005 and $31^{\text {st }}$ December 2017, at the Charlotte Maxeke Johannesburg Academic Hospital Trauma Unit. The information was extracted from the Digital Patient Registry of the Unit, clinical notes and theatre registry. Data selected for analysis included: age and gender; mechanism of injury, haemodynamic status (heart rate, respiratory rate, systolic blood pressure-sBP, Glasgow Coma Score (GCS); oesophageal temperature (measured in operating theatre once patient arrived there); coded Revised Trauma Score (cRTS) and Injury Severity Score (ISS); primary anatomical area of injury; arterial blood gas results if obtained, and 24hour mortality.

The data extracted was analyzed with the Statistical Package for the Social Sciences (SPSS) software, version 21.0 (Mac OS) from IBM Corporation (Armonk, NY, USA) under a temporary license obtained from www.ibm.com/ sofware/analytics/spss/

The analysis included all patients whose records were available, further analysis was done between survivor and non-survivor sub-groups. Student $T$ test and Chi squared $\left(X^{2}\right)$ test were used to establish significance, a value of $\mathrm{p}<$ 0,05 was considered significant.

The Human Research Ethics Committee of the University of Witwatersrand granted permission for the study (M180501).

\section{Results}

One hundred and ten patients underwent ERT during the study period. One-hundred and one were males (92\%) and 9 females; the median age was 29 years (Inter Quartile Range - IQR 16-58). Of the total number of patients, 61 sustained stab wounds $(55.5 \%), 43$ sustained gunshot wounds-GSW (39\%) and 6 were blunt trauma victims $(5.5 \%)$.

Overall, the physiological parameters were recorded as follows: Heart Rate (HR) 84 beats per minute (IQR 0-154); Systolic Blood Pressure (SBP) $60 \mathrm{mmHg}$ (IQR 0-104); Respiratory Rate (RR) 10 breaths per minute (IQR 0-56) and Glasgow Coma Score 8. (Table 2)

Only 55 of the 110 patients had a complete arterial blood gas analysis recorded in the emergency department. The mean $\mathrm{pH}$ was 7.16 (IQR 6,5-7,65); Base Excess -15,7 (IQR -3 to -31) and serum Lactate $12.4 \mathrm{mmol} / \mathrm{L}$ (IQR 2.422) denoting the severe metabolic acidosis that accompanies major trauma.

Majority of the patients were hypothermic during and after the ERT, median Temperature was $35^{\circ}$ Celsius (IQR

Table 1 Demographics

\begin{tabular}{|l|c|c|c|}
\hline & All $\mathrm{n}=110,(\%, \mathrm{IQR})$ & Survivors $\mathrm{n}=24,(\%, \mathrm{IQR})$ & Non-survivors $\mathrm{n}=86,(\%, \mathrm{IQR})$ \\
\hline Male & 101 & 22 & 79 \\
Female & 9 & 2 & 7 \\
\hline Median Age & $29(16-58)$ & $28(17-36)$ & $29,2(16-58)$ \\
\hline Stabs & $61(55.4)$ & $20(18.1)$ & $41(37.2)$ \\
GSW & $43(39)$ & $4(3.6)$ & $39(35.5)$ \\
Blunt & $6(5.4)$ & 0 & 6 \\
\hline
\end{tabular}

IQR: inter quartile range; GSW gunshot wound 
Table 2 Comparative Physiological and Metabolic Variables

\begin{tabular}{|l|l|l|}
\hline Variable & Survivor n=24 (IQR) & Non-survivor n=86 (IQR) \\
\hline Systolic BP mmHg & $57(0-98)$ & $48(0-104)$ \\
\hline Respiratory Rate & $13(0-45)$ & $11(0-56)$ \\
\hline GCS & 8 & 7 \\
\hline Heart Rate & $97(0-154)$ & $81(0-154)$ \\
\hline Temperature 0C (Oesophageal measured in OR) & $35,3(35-36,5)$ & $35(32-37)$ \\
\hline pH & $7.16(7.34-6.67)$ & $7.16(7.38-6.50)$ \\
\hline Base Deficit & $-15.8(-24$ to -6.9$)$ & $-16(-31$ to -3$)$ \\
\hline Serum Lactate & $10.3(2.4-15)$ & $13(4.7-22)$ \\
\hline cRTS & $5,6(0-12)$ & $4,2(0-11)$ \\
\hline ISS & $24(10-29)$ & $27(16-59)$ \\
\hline
\end{tabular}

IQR: inter quartile range; BP: blood pressure; GCS: Glasgow coma score, cRTS: calculated revised trauma score, ISS: injury severity score, OR: operating room

32-37). The oesophageal temperature measurement was obtained in the operating room after the patients were moved there to complete the procedure, as part of the anaesthesia protocol for trauma patients.

Comparing the results, there was only a minor difference in the serum Lactate levels in favor of the survivors $(10,3 \mathrm{mmol} / \mathrm{L}$ versus $13 \mathrm{mmol} / \mathrm{l})$. However, none of the physiological and metabolic parameters were statistically significant when comparing survivors with nonsurvivors. Table 2

Overall, the mean ISS was 25 (IQR 16-59) and RTS was 4,5 (IQR 0-12) with an overall probability of survival of $50.4 \%$. The mean ISS of the group of survivors was 24 (IQR 10-29) versus 27 (IQR 16-59) for the non-survivors, not statistically significant results.

Most patients received crystalloids (Ringer Lactate) as part of the initial resuscitation protocol; the mean volume administered to the survivors was $1625 \mathrm{ml}$ (IQR 0-3000 ml) versus $1550 \mathrm{ml}$ (IQR 0 to $4000 \mathrm{ml}$ ) for the non-survivors. Nearly $40 \%$ of patients also received a synthetic colloid infusion, the mean volume was $479 \mathrm{ml}$ (IQR 0-3000 $\mathrm{ml}$ ) for survivors versus $1027 \mathrm{ml}$ (IQR 0 to $9000 \mathrm{ml}$ ) for non-survivors. Seventy-three patients $(66.3 \%)$ received transfusions during the ERT; survivors received a median of 2,3 units of Packed Red Cells, to a maximum of 10 units during the resuscitation versus 3,2 units for the nonsurvivors (maximum 13 units)

The survivors received 1 unit of Fresh Frozen Plasma compared to 2 units for non-survivors. The volume and type of fluids used during the ERT did not showed a statistically significant impact in mortality.

ERT were performed for injuries in the thorax $(n=65)$, abdomen $(n=17)$, thoraco-abdominal $(n=25)$ and pelvis and femoral vessels $(n=3)$. Six patients had ERT for blunt trauma. The overall survival of the series was $21,8 \%$ (40.6 $\%$ survival for cardiac injuries; $20 \%$ for thoracic injuries and $1.8 \%$ for injuries below the diaphragm). Patients with stab wounds have better survival $(32.8 \%)$ that those with GSW $(9.3 \%)$ or blunt trauma (0\%).
Comparing isolated chest trauma $(n=65)$ to injuries elsewhere in the torso the difference in survival is significant: Thorax alive $\mathrm{n}=22$ versus other areas alive $\mathrm{n}=2(p=0.001)$ Cardiac injuries were present in $29 \%$ of patients $(n=32)$ resulting in the best outcome: Alive $\mathrm{n}=13(40.6 \%)(p=$ 0.032).

Table 3 Comparative Outcome by Injured Region

\begin{tabular}{|l|l|l|}
\hline Anatomical Region & Survivor: $\mathrm{n}(\%)$ & Non-survivor: $\mathrm{n}(\%)$ \\
\hline Chest $\mathrm{n}=65$ & $22(20)(\mathrm{p}=0.001)$ & $43(80)$ \\
\hline Abdomen $\mathrm{n}=17$ & 1 & 16 \\
\hline Thoraco-abdominal $\mathrm{n}=25$ & 1 & 24 \\
\hline Pelvic-femoral $\mathrm{n}=3$ & 0 & 3 \\
\hline Heart $\mathrm{n}=32(29 \%)$ & $13(40.6)(\mathrm{p}=0.032)$ & $19(56.5)$ \\
\hline
\end{tabular}

Two patients survived the ERT when performed for injuries elsewhere in the torso, one patient with abdominal GSW and one with thoraco-abdominal GSW. No other patient survived an ERT for injuries outside the chest.

Figure 1 Outcomes Anatomical Region/Organ/Mechanism of Injury

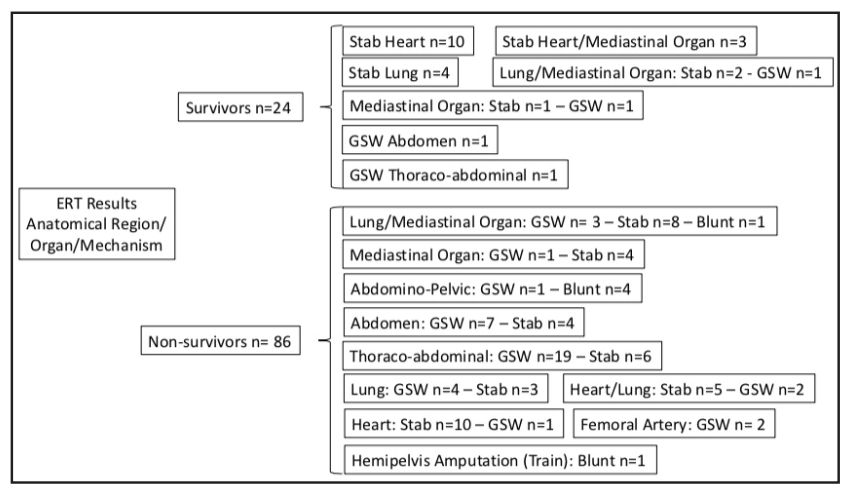




\section{Discussion}

Patients arriving to the Trauma Unit at Charlotte Maxeke are transported by ambulance services and occasionally by private vehicles. The level of care in the ambulances varies among services, with some of the ambulances manned by Emergency Medical Technicians and others by basic rescue providers. Unfortunately, communication between prehospital rescue personnel and the base hospital is limited, seldom the condition of the patient is known before arrival.

In most South African Trauma Centres an ERT is performed to assist with the resuscitation of patients with cardiac tamponade or exsanguination from penetrating torso trauma. The use of ERT for blunt trauma victims remains controversial due to the accompanying high mortality and limited availability of resources in state funded hospitals.

Most of the international literature of the late 1990's and early 2000's [13-31] discouraged the use of ERT in blunt trauma, however there are recent studies of the use of ERT in blunt trauma with acceptable success. [32, 33]

In our literature review we did not find any reports of the use of metabolic parameters to help selecting the patient for ERT, our hypothesis was that patients with penetrating torso injuries below the diaphragm were presenting with severe metabolic derangement similar to the one exhibited by blunt trauma victims, therefore incurring in high mortality despite ERT. As shown by our results the metabolic derangement was important but not statistically significant between the survivor or non-survivors.

The overall mortality of the series was 78,2\%. The available literature reports mortality above 50\% for penetrating torso trauma and near $100 \%$ for blunt injuries, however a recent systematic review from Scandinavia showed encouraging results for blunt trauma victims. [13-33]

In 1991, Ivatury et al [19] reported an overall survival of $9.8 \%$ following ERT (24.5\% Stab vs. $4.7 \%$ GSW vs. Nil in Blunt trauma), they demonstrated that best results are achieved in patients with vital signs on arrival and those with a potential cardiac injury. Asensio et al [14] in 1998 reported an overall survival of $16 \%$ for patients following ERT for penetrating cardiac injury, in whom, determinants of survival were the presence or absence of sinus rhythm, mechanism of injury (Stab vs. GSW), need for aortic crossclamping and inability to restore organized rhythm or Blood Pressure after the thoracotomy. In 2004, an analysis of 42 series of patients (7,035 patients including children) found that $11.1 \%$ survived penetrating injuries vs. $1.6 \%$ blunt, with an overall mortality rate of $92.2 \%$. [20]

It should be noted that the use of synthetic colloids has been eliminated in our unit and the initial crystalloid use is limited, with early blood product administration according to the current guidelines for the management of bleeding trauma patients [2-11]. It could be of interest to assess the impact of this change in fluid management strategy in the subgroup of patients requiring ERT under these conditions.

The overall survival of the series was $21,8 \%$, further analysis combining mechanism of injury, anatomical zones and mortality revealed statistically significant better survival $(p=0.032)$ for penetrating cardiac trauma. Penetrating chest trauma in general (cardiac, lung and mediastinal) had a statistically significant $(p=0.001)$ lower mortality than the rest of the combined anatomical areas, irrespective of mechanism. These results correlate well with the available literature [1,13-34]

It is self-explanatory that a patient with a (potential) cardiac injury will benefit from rapid evacuation of the tamponade and control of the cardiac laceration, therefore these patients are the ideal candidates for ERT, as long as there are signs of cardio-respiratory activity (breathing effort, palpable pulse, blood pressure and organized cardiac rhythm) or a witnessed cardiac arrest on arrival to ED. Same rationale applies to the rapid exsanguination or air embolism secondary to penetrating mediastinal and pulmonary trauma.

Barring the obvious limitations of a retrospective review our results suggest that the traditional indications to use ERT could be adjusted, especially in a resourcesconstrained environment. In patients with blunt injuries and penetrating thoraco-abdominal or abdomino-pelvic trauma, the degree of physiologic and metabolic deterioration and the magnitude of the injuries significantly reduce the possibility of survival. In these situations, the decision to perform an ERT should be individualized. Despite the lack of statistical power of our results, we believe that the severity of injury expressed as physiological and metabolical derangement that can be demonstrated by immediate point of care blood gas analysis $(\mathrm{pH}$, Base Deficit, Lactate) could still play a significant role in predicting negative outcomes in patients undergoing ERT.

Further studies to include these variables in the decision-making process may give us a better answer.

\section{Conclusions:}

In a resource constrained environment an Emergency Room Thoracotomy should be performed in patients with a thoracic injury, especially cardiac, to achieve the best possible outcome.

COI Statement: This paper has not been submitted in parallel. It has not been presented fully or partially at a meeting or podium or congress. It has not been published nor submitted for consideration beforehand.

This research received no specific grant from any funding agency in the public, commercial, or nonprofit sectors. There are no relevant or minor financial relationships from authors, their relatives or next of kin with external companies.

Disclosure: The authors declared no conflict of interest. No funding was received for this study. 


\section{References}

1. Biffl WL, Moore EE, Johnson JL: Emergency Department thoracotomy (2004) In Moore EE; Feliciano DV; Mattox KL (eds.) Trauma, McGraw Hill, New York, p. 239-252

2. Spahn DR, Bouillon B, Cerny V, et al (2019) The European guideline on management of major bleeding and coagulopathy following trauma: fifth edition Critical Care 23:98-172

3. Brohi K, Cohen MJ, Ganter MT, et al (2008) Acute Coagulopathy of Trauma: hypoperfusion induces systemic anticoagulation and hyperfibrinolysis. J Trauma 64:12111217

4. Brohi K, Frith D (2012) The pathophysiology of traumainduced coagulopathy. Curr Opin Crit Care 18:631-636

5. Holcomb JB, Wade CE, Michalek JE, et al (2008) Increased plasma and platelet to red blood cells ratios improves outcome in 466 massively transfused civilian trauma patients. Ann Surg 248:447-58

6. Holcomb JB, Zarzabal LA, Michalek JE, et al (2011) Increased platelet: RBC ratios are associated with improved survival after massive transfusion. J Trauma 71(2 Suppl 3):S318-328

7. Brown LM, Aro SO, Cohen MJ, et al (2011) A high fresh frozen plasma: packed red blood cell transfusion ratio decreases mortality in all massively transfused trauma patients regardless of admission international normalized ratio. J Trauma 71(2 Suppl 3):S358-363

8. Borgman MA, Spinella PC, Perkins JG, et al (2007) The ratio of blood products transfused affects mortality in patients receiving massive transfusion at a combat support hospital. J Trauma 63:805-813

9. Davenport R, Curry N, Manson J, et al (2011) Hemostatic effects of fresh frozen plasma may be maximal at red cell ratios of 1:2. J Trauma 70:90-95

10. Cotton BA, Brigham K, Nunez TC, et al (2009). Predefined massive transfusion protocols are associated with a reduction in organ failure and postinjury complications. J Trauma 66:41-48

11. Cohen MJ (2012) Towards hemostatic resuscitation: the changing understanding of acute traumatic biology, massive bleeding and damage-control resuscitation. Surg Clin North Am 92:877-891

12. Blachford JW III (1985) Ludwig Rehn - The first successful cardiorrhaphy. Ann Thorac Surg 39:492-495

13. Moore EE, Knudson MM, Burlew CC, et al. (2011) Defining the limits of resuscitative emergency department thoracotomy: a contemporary Western Trauma Association perspective. J Trauma 70:334-339

14. Asensio JA, Murray J, Demetriades D, et al (1998) Penetrating cardiac injuries: a prospective study of variables predicting outcomes. J Am Coll Surg 186:24-34

15. Velmahos GC, Degiannis E, Souter I, et al (1995) Outcome of a strict policy on emergency department thoracotomies. Arch Surg 130: 774-777

16. Campbell NC, Thompson SR, Muckart DJ, et al (1997) Review of 1198 cases of penetrating cardiac trauma. Br J Surg 84:1737-40

17. Bowley DMG, Degiannis E, Smith MD. Penetrating Chest Injuries (2004) In: Nicol A, Steyn E (eds.) Oxford Handbook of Trauma for Southern Africa. Oxford University Press, Cape Town, p. 208-223

18. Powell DW, Moore EE, Cothren CC, et al (2004) Is emergency department resuscitative thoracotomy futile care for the critically injured patient requiring prehospital cardiopulmonary resuscitation? J Am Coll Surg 199:211-215

19. Ivatury RR. (1991) "Directed" emergency room thoracotomy: a prognostic prerequisite for survival. J Trauma 31:1076-1081

20. Asensio JA, O'Shanahan G, Petrone P, et al (2004). Emergency thoracotomy: a critical evaluation of the technique Cir Gen 26:128-137

21. Brown SE, Gomez GA, Jacobson LE, et al (1996) Penetrating chest trauma : should indications for emergency room thoracotomy be limited ? Am Surg 62:530-533

22. Ivatury RR, Shah PM, Ito K, et al (1981) Emergency room thoracotomy for the resuscitation of patients with "fatal" penetrating injuries of the heart. Ann Thorac Surg 32:377-385

23. Esposito TJ, Jurkovich GJ, Rice CL, et al (1991) Reappraisal of emergency room thoracotomy in a changing environment. J Trauma 31:881-85

24. Asensio JA, Petrone P, Roldan G et al (2003) Analysis of 185 Iliac Vessel Injuries: Risk factors and predictors of outcome. Arch Surg 138:1187-1193

25. Working Group, Ad Hoc Subcommittee on Outcomes, American College of Surgeons-Committee on Trauma (2001) Practice management guidelines for emergency department thoracotomy. J Am Coll Surg 193:303-309

26. Rhee PM, Acosta J, Bridgeman A, et al (2000) Survival after emergency department thoracotomy: review of published data from the past 25 years. J Am Coll Surg 190:288-299

27. Seamon MJ, Pathak AS, Bradley KM, et al (2008) Emergency department thoracotomy: still useful after abdominal exsanguination? J Trauma 64:1-7

28. Onat S (2010) Urgent thoracotomy for penetrating chest trauma: analysis of 158 patients of a single center. Injury 41:876-880

29. Gomez G, Fecher A, Joy T, et al (2010) Optimizing outcomes in emergency room thoracotomy: a 20-year experience in an urban Level I trauma center. Am Surg 76:406-410

30. Seamon MJ, Shiroff AM, Franco M, et al (2009) Emergency department thoracotomy for penetrating injuries of the heart and great vessels: an appraisal of 283 consecutive cases from two urban trauma centers. J Trauma 67:1250-1257

31. Burlew CC, Moore EE, Moore FA, et al (2012) Western Trauma Association critical decisions in trauma: resuscitative thoracotomy. J Trauma Acute Care Surg 73:1359-1363

32. Pahle AS, Pedersen BL, Skaga NO, et al (2010) Emergency thoracotomy saves lives in a Scandinavian hospital setting. J Trauma 68:599-603

33. Narvestad JK, Meskinfamfard M, Søreide K. Emergency resuscitative thoracotomy performed in European civilian trauma patients with blunt or penetrating injuries: a systematic review. Eur J Trauma Emerg Surg 2016;42(6):677-685

34. Keller D, Kulp H, Maher Z, et al (2013) Life after near death: long-term outcomes of emergency department thoracotomy survivors. J Trauma Acute Care Surg 74:1315-1320

35. Aso S, Matsui H, Fishimi K, t al (2017) Resuscitative endovascular balloon occlusion of the aorta or resuscitative thoracotomy with aortic clamping for noncompressible torso hemorrhage: A retrospective nationwide study J Trauma Acute Care Surg 82: 910-914

36. Moore LJ, Brenner M, Kozar R, et al (2015) Implementation of resuscitative endovascular balloon occlusion of the aorta as an alternative to resuscitative thoracotomy for noncompressible truncal haemorrhage, Acute Care Surg 79: 523-532 